\title{
Educación en tiempos de pandemia: reflexiones de alumnos y profesores sobre la enseñanza virtual universitaria en España, Italia y Ecuador
}

\author{
Education in times of pandemic: reflections of students and \\ teachers on virtual university education in Spain, Italy and Ecuador
}

Santiago Tejedor. Universidad Autónoma de Barcelona. España.

santiago.tejedor@uab.cat

$[\mathrm{CV}]$ G

Laura Cervi. Universidad Autónoma de Barcelona. España.

laura.cervi@uab.cat

$[\underline{\mathrm{CV}}]$ (D) $\mathrm{R}^{\mathrm{C}}$

Fernanda Tusa. Universidad Técnica de Machala. Ecuador.

ftusa@utmachala.edu.ec

$[\mathrm{CV}]$ (1) $\mathrm{G}$

Alberto Parola. Università degli Studi di Torino. Italia.

alberto.parola@unito.it

$[\mathrm{CV}]$ (1) $\mathrm{R}^{\mathrm{C}}$

Cómo citar este artículo / Referencia normalizada

Tejedor, S., Cervi, L., Tusa, F. y Parola, A. (2020). Educación en tiempos de pandemia: reflexiones de alumnos y profesores sobre la enseñanza virtual universitaria en España, Italia y Ecuador. Revista Latina de Comunicación Social, 78, 1-21. https://www.doi.org/10.4185/RLCS$\underline{2020-1466}$

\section{RESUMEN}

Introducción. Esta investigación realizó un estudio comparativo entre tres países muy impactados por el coronavirus a partir del análisis de las reflexiones de docentes y estudiantes sobre la enseñanza virtual universitaria durante la etapa de confinamiento. Metodología. El estudio, de carácter descriptivo, exploratorio y explicativo, aplicó encuestas, entre marzo y abril de 2020, a estudiantes y docentes de Periodismo, Comunicación y Educación de la Universidad Autónoma de Barcelona (España), Universidad de Torino (Italia) y Universidad Técnica de Machala (Ecuador). La encuesta tuvo respuestas de 300 estudiantes (100 por país) y 196 docentes. Resultados. Los estudiantes, de forma mayoritaria, valoran negativamente el paso a la virtualidad, pues este se asocia, de forma recurrente, con un incremente de la carga lectiva. La teleformación ha contribuido a impactar negativamente en la visión del alumnado sobre sus formadores, mientras que en estos últimos demandan competencias digitales básicas en los jóvenes universitarios. Discusión. Según los hallazgos obtenidos, los docentes, en el escenario de la teleformación, deben mostrar conocimientos no solo sobre el contenido de la materia, sino también conocimiento tecnológico y pedagógicodigital. Conclusiones. El docente tiene que ser capaz de innovar, reflexionar y transformar sus 
propuestas didácticas para responder a las demandas sociales que vive el mundo en medio de una crisis sanitaria, a la vez que se alcancen los objetivos curriculares propuestos al inicio del curso. Por otro lado, los docentes encuestados reconocen que es necesario la promoción del pensamiento crítico y reflexivo vinculado a la gestión estratégica de las TIC.

PALABRAS CLAVE: Tecnología educativa; Educación Superior; Educación pública; proceso educativo; adaptación estudiantil; educación a distancia, aprendizaje virtual.

\begin{abstract}
Introduction. This research carried out a comparative study between three countries severely impacted by the coronavirus based on the analysis of the reflections of teachers and students on university virtual teaching during the confinement stage. Methodology. This descriptive, exploratory and explanatory study applied surveys, between March and April 2020, to students and teachers of Journalism, Communication and Education at the Autonomous University of Barcelona (Spain), University of Torino (Italy) and Technical University from Machala (Ecuador). The survey had responses from 300 students (100 per country) and 196 teachers. Results. The majority of students negatively value the move to virtuality, since it is associated, on a recurring basis, with an increase in the teaching load. E-learning has contributed to negatively impacting the view of students on their trainers, while the latter demand basic digital skills in young university students. Discussion. According to the research results, teachers, in the scenario of remote training, must show knowledge not only about the content of the subject, but also technological and digital-pedagogical knowledge. Conclusions. The teacher must be able to innovate, reflect and transform their didactic proposals to respond to the social demands that the world is experiencing in the midst of a health crisis, while achieving the curricular objectives proposed at the beginning of the course. On the other hand, the surveyed teachers recognize that it is necessary to promote critical and reflective thinking linked to the strategic management of ICT.
\end{abstract}

KEYWORDS: Educational technology; Higher Education; public education; educational process; student adaptation; distance education; virtual learning.

\title{
CONTENIDO
}

1. Introducción. 2. Marco Teórico. 3. Objetivos. 4. Metodología. 5. Resultados. 6. Discusión. 7. Conclusiones. 8. Bibliografía.

\section{Introducción}

En los últimos tiempos, las sociedades han cambiado de forma precipitada. De hecho, teóricos como Harnad (1991) consideran que la historia de la humanidad está en dependencia de la evolución tecnológica, lo que ha dado lugar a cambios radicales en la organización del conocimiento y en la cognición humana. En este contexto, el acceso a Internet ha permitido elevar la calidad de vida y la prosperidad de un país (Olivares Carmona et al., 2018). Por ello se advierte que su uso es indispensable para favorecer la comunicación, el aprendizaje y la cultura, propiciando a que los ciudadanos estén mejor preparados y sean más competitivos (Vera, Torres y Martínez, 2014).

Fruto del proceso de globalización, la accesibilidad, la interactividad y la flexibilidad de las Tecnologías de la Información y la Comunicación (TIC) ha llevado a un mayor uso de internet, redes sociales y videojuegos, cambiando nuestra manera de trabajar, pensar y tomar decisiones (Garrote, Jiménez y Serna, 2018). Esto ha repercutido en el rol y en las características medulares de las instituciones educativas (López Catalán et al., 2018). Como resultado, la educación se ha visto 
afectada de forma radical por la emergencia de la cultura digital, provocando la imperiosa necesidad de innovar (Freire, 2009).

En este escenario, las universidades deben ofrecer una Educación Superior que forme sujetos altamente competentes para afrontar los complejos desafíos de la ciencia, la economía y las relaciones sociales (Area, 2010). Ello justifica por qué las TIC están adquiriendo un fuerte protagonismo en los nuevos contextos formativos (Cabero Almenara, 2014), puesto que la escuela no debe ser ajena a las transformaciones que las TIC están provocando y ha de adaptarse a las formas emergentes de aprender de las generaciones centennials (Pérez-Escoda, Castro-Zubizarreta y FandosIgado, 2016).

Tal y como se analiza, los cambios tecnológicos han supuesto un aumento de oportunidades en el área de la formación e-learning, donde nuevos modelos, metodologías, recursos y prácticas han dado lugar a diversas tendencias identificadas en la literatura, como la utilización de aula virtual (LMS), cursos MOOC, Open Course Ware (OCW), cursos en abierto, repositorios institucionales, mobile learning, gamificación, social media y realidad aumentada (Bozkur et al., 2015; Davies et al., 2010).

El e-learning se presenta entonces como una estrategia formativa generalizada para afrontar diversas problemáticas educativas y las demandas de perfeccionamiento continuo de la Sociedad de Conocimiento, dado su ahorro económico, gestión del tiempo y componente de interactividad (Martínez-Caro, 2008). De modo complementario, autores como Aguaded y Díaz (2009) enfatizan en cinco usos que puede tener Internet en el contexto de la universidad, como son: 1) presencia institucional en los nuevos espacios de comunicación social, 2) gestión a través de la red de cuestiones administrativas, 3) utilización de los recursos telemáticos con fines de investigación, 4) acceso virtual a los fondos bibliográficos y 5) espacio para la docencia apoyada en redes de ordenadores, para fines educativos.

Para que la teleformación funcione, es fundamental conocer de antemano las creencias que los profesores tengan respecto al papel que las TIC puedan desempeñar en la enseñanza (Chen 2008; Teo, 2008), puesto que la poca alfabetización digital del profesorado dificulta la utilización técnicodidáctica de las TIC (Valdés et al., 2010; Ballestero et al., 2010). En esta línea, Hammond et al. (2009) advierten que la formación docente en TIC es un elemento determinante en su práctica de la enseñanza. Sin embargo, las investigaciones de Valerio y Paredes (2008) y Goktas et al. (2008) demuestran que los docentes son capacitados en un manejo instrumental de las TIC y no en un uso socioconstructivo de la tecnología educativa.

La COVID-19 ha evidenciado la urgente transformación que demandan los sistemas educativos tradicionales y la importancia de poseer una estrategia educativa virtual, así como un alumnado y un profesorado con habilidades y competencias para la enseñanza y el aprendizaje en el ciberespacio. La irrupción de este virus planetario ha dejado constancia de las exigencias de una pandemia a las instituciones educativa: flexibilidad, plataformas, metodologías y contenidos adaptados a unos intercambios formativos mediados por las pantallas. En este sentido, esta investigación desarrolla un estudio comparativo entre tres países muy impactado por el coronavirus a partir del análisis de las reflexiones de docentes y estudiantes sobre la enseñanza virtual universitaria durante la etapa de confinamiento.

\section{Marco Teórico}

Aunque es una crisis sanitaria, la universidad pública también ha padecido las consecuencias de la pandemia mundial de la COVID-19. En este sentido, ha tenido que afrontar un cambio repentino, 
moviéndose de la presencialidad a la no presencialidad. Esta educación, mediada por tecnología, ha solicitado de un esfuerzo de aprendizaje exprés para muchos docentes (Cáceres-Muñoz, Jiménez Hernández y Martín-Sánchez, 2020). Como resultado, los contextos mediados por un acceso a la educación a distancia, a través de tecnologías digitales, han sido desiguales; en particular, para los grupos sociales de mayor vulnerabilidad (Cervantes Holguín y Gutiérrez Sandoval, 2020).

En este escenario, las administraciones educativas deben velar porque todo alumnado tenga acceso a la misma y en las mismas condiciones (Bekerman y Rondanini, 2020; Cotino Hueso, 2020), ya que se ha puesto énfasis en la transformación de la cultura educativa por una cultura educativa digital en la que ni todos ni todas cuentan con las mismas posibilidades (Beltrán y Venegas et al., 2020). En efecto, esta presencialidad remota ha dejado de considerar las particularidades y las condiciones de accesibilidad para garantizar la inclusión, la equidad y la calidad en la formación universitaria (Fontana-Hernández, Herrera-Sibaja, Leiva-Durán, Montero-Cascante, 2020). Como secuela, las perspectivas de un futuro indeterminado son un pensamiento con el que gran parte de la población estudiantil ha tenido que convivir en esta época (Cao et al., 2020).

La suspensión de las actividades docentes presenciales para dar paso a la virtualización ha supuesto que el profesorado y el alumnado tengan una elevada presión, tanto en su trabajo como en la adaptación al nuevo escenario. Además, la transición a esta docencia online ha sido abrupta, desconociendo los largos procesos de formación, de rediseño de asignaturas y de infraestructuras que soporten la demanda de la tele-enseñanza (González-Calvo, Barba-Martín, Bores-García, GallegoLema, 2020). De hecho, en varios países latinoamericanos, la COVID-19 influyó negativamente en el proceso de enseñanza-aprendizaje, debido a que no se invirtió durante muchos años en la adecuación de los campus virtuales, los sitios web institucionales, las revistas científicas digitales y en la capacitación de los docentes y alumnos en el manejo de las TICs, teniendo que improvisar soluciones tecnológicas (Ríos Campos, 2020).

Por su parte, los estudiantes de zonas rurales no han podido mantenerse al día con este modelo de enseñanza virtual, puesto que en sus hogares el acceso a Internet es limitado y la señal del teléfono es pobre (Carla Silva, Ramos Silva y Montanari, 2020). Otro informe revela que incluso cuando las familias tienen un teléfono celular, la situación de insuficiencia permanece, ya que hay varios estudiantes dentro de casa que exigen el uso de teléfonos celulares, lo que hace imposible que todos cumplan con sus compromisos académicos (Aquino et al., 2020; Correa Filho \& Segall-Corrêa, 2020). Además, la educación remota en las zonas rurales se enfrenta al problema estructural del hogar, donde los estudiantes no tienen un ambiente adecuado para estudiar (Tarcízio, 2020; Tenente, 2020).

Este tránsito hacia un nuevo paradigma de enseñanza-aprendizaje ha visibilizado las desigualdades en el desarrollo educativo bajo las actuales condiciones de crisis pandémica, siendo la cooperación con instituciones, empresas y ONG la respuesta para apoyar a las instituciones educativas que carecen de capacidades, recursos y medios para llevar con éxito la educación a distancia (Quevedo Ramírez, 2020). Y es que la educación mediada por tecnologías en tiempos de la COVID-19 no solo debe centrase en los estudios académicos, sino en el cuidar al estudiante que enfrenta la fragilidad de la vida y poner al ser como centro de toda actividad humana (Monasterio y Briceño, 2020).

Ante esta situación de excepcionalidad educativa, el docente debe ser soporte, promotor de la resiliencia, guía en lo académico, animador, asesor emocional y garante de la organización y coherencia institucionales (Villafuerte et al., 2020). Al respecto, los embates de la COVID-19 han redefinido nuevas líneas de acción educativa donde el proceder de los docentes ha privilegiado la enseñanza de aquellos conocimientos que representan un aporte valioso para la vida cotidiana, sin 
dejar de lado la comprensión empática con el estudiante (Morales, 2020). Para Herrera, Tusa y Maza (2019), no se trata de que el alumno se adapte y se integre, sino que la universidad apueste por él y haga todo lo necesario para conseguir que tenga una educación de calidad.

La COVID-19 invita a una revisión pedagógica y a la reestructuración de la oferta formativa en la Educación Superior. Es más, muchas instituciones han emprendido el camino hacia una renovación de contenidos que favorezca tanto la calidad como la equidad (Pedró, 2020). La pandemia se presenta entonces como una oportunidad de reflexionar, junto con los estudiantes, acerca de nuestro rol en tiempo de crisis como personas, como ciudadanos y como profesionales (Millán, Heresi, Díaz, Weisstaub y Vargas, 2020).

La crisis pandémica, trasladada al campo educativo, muestra los profundos cambios que requieren los programas de formación y las competencias que demandan los profesionales del futuro. La educación de calidad es más necesaria que nunca para la promoción de una nueva generación de graduados que haga frente a los múltiples desafíos del siglo XXI, jóvenes que trabajen hacia una distribución más equitativa del bienestar y conviertan a la sociedad en un lugar más habitable y pacífico, no solo para unos pocos, sino para todos (Feyen, 2020).

\section{Objetivos}

Analizar la percepción del alumnado y del cuerpo docente de las carreras de grado de Comunicación, Periodismo y Educación de universidades públicas de Ecuador (Universidad Técnica de Machala), Italia (Universidad de Torino) y España (Universidad Autónoma de Barcelona) acerca de la enseñanza virtual durante la crisis sanitaria de la COVID-19, mediante una encuesta online aplicada entre marzo y abril de 2020.

\section{Metodología}

Esta investigación, de carácter descriptivo, exploratorio y explicativo, aplicó un estudio comparativo entre España, Italia y Ecuador sobre la docencia virtual impuesta durante la crisis del coronavirus. La propuesta metodológica se diseñó a partir de una perspectiva exploratoria (Vilches, 2011) y aplicó el instrumento de la encuesta, dirigida a estudiantes y docentes de Periodismo, Comunicación y Educación de los tres países seleccionados. La encuesta tuvo respuestas de 300 estudiantes (100 por país) y 196 docentes. Las encuestas se desarrollaron entre marzo y abril de 2020.

La muestra invitada, concebida como el conjunto de elementos de la población a los que se pide que participen en la investigación (Del Rincón, Arnal, Latorre y Sans, 1995), corresponde a estudiantes y docentes, estableciéndose una muestra aleatoria simple. Tal y como apuntan Colás et al. (2013), el estudio consideró la encuesta online como mecanismo metodológico óptimo para recoger información, y se direccionó a estudiantes de diferentes franjas de edad (entre 18 y 28 años), siendo 21 años con un $37,6 \%$ el más numeroso en el total de la muestra. El porcentaje analizado se considera significativo dentro del universo y, en este sentido, la muestra permite inferir resultados precisos.

En España, la participación ha sido de 71,3\% de mujeres y 28,7\% de hombres, cuyas edades oscilan entre 18 a 23 años. En Italia, la participación ha sido del 84,37\% de mujeres y del 16\% de hombres, cuyas edades oscilan entre los 16 y los 30 años, concentrándose principalmente en edades comprendidas entre los 19 y 27 años. En el caso de Ecuador, han participado un 64,4\% de mujeres y un $35,6 \%$ de hombres, con edades comprendidas entre los 18 y los 28 años. Respecto a los docentes 
consultados, la encuesta se ha direccionado a 196 docentes universitarios, de los cuales $23 \%$ son doctores, $50,5 \%$ tienen título de máster, $13 \%$ son licenciados y 13,5 cuentan con otro tipo de estudio.

La investigación se ha basado en un muestreo discrecional o muestreo intencional en la selección de las tres universidades participantes. En este tipo de toma de muestras, los sujetos sometidos a análisis y estudio son elegidos por el investigador para formar parte de la muestra con un objetivo específico (Vilches, 2011) al ser considerados como adecuados o idóneos para la investigación. Se han escogido tres universidades de escenarios socio-geográficos que han recibido un fuerte impacto del virus COVID-19, generando una cifra de afectados y fallecidos muy elevada dentro de su entorno territorial. Al respecto, las universidades que integran el estudio son las siguientes:

- Universidad Autónoma de Barcelona (España): Está considerada como una de las mejores universidades europeas (en investigación y docencia), según la mayoría de directorios académicos universitarios europeos. El 26 de noviembre del 2009, la UAB obtuvo el reconocimiento de Campus de Excelencia Internacional. Cuenta con 37.166 alumnos y 3.262 profesores. La UAB ofrece 77 titulaciones de grado, 328 programas de postgrado y 90 programas de doctorado. El estudio ha contado con la participación de los estudiantes del grado de Periodismo.

- Universidad de Torino (Italia). Es una universidad pública, fundada en 1404, que se encuentra entre las más antiguas de Europa. El estudio ha contado con la participación de los estudiantes del grado de Educación.

- Universidad Técnica de Machala (Ecuador): Es una Institución de Educación Superior, de carácter público, ubicada en la provincia de El Oro, al sur del país. Pese a encontrarse en una zona de vulnerabilidad fronteriza, ha conseguido acreditarse en categoría B como universidad de excelencia. Cuenta con cinco facultades y cerca de 13.000 estudiantes están inscritos en su oferta académica, tanto de pregrado como de posgrado. El estudio ha contado con la participación de los estudiantes del grado de Periodismo.

El estudio adoptó una metodología de trabajo híbrida a partir de una encuesta matriz integrada por cuestiones de tipo cuantitativo y preguntas abiertas para posibilitar igualmente un trabajo de índole cualitativa. La encuesta se ha diseñado con preguntas cerradas de escalas nominales excluyentes y se ha basado en un muestreo aleatorio simple sin reposición.

El análisis estadístico empleado sobre la encuesta ha sido el análisis descriptivo basado en frecuencias y porcentajes. Las respuestas totales de cada país, diferentes en cada caso, se han equiparado en porcentajes con una intención comparativa que ha permitido un cruce final entre los tres países seleccionados. Además, la propuesta metodológica incluye preguntas cerradas dicotómicas para los temas más definidos sobre el uso, la valoración de las plataformas y el impacto de las redes sociales en desempeño cotidiano y profesional. Se ha considerado pertinente incorporar preguntas categorizadas (ofreciendo al encuestado una serie de listados de posibles actividades a desarrollar en una red social o diferentes nombres de plataformas dialógicas on-line) y, de este modo, se han combinado preguntas de identificación, de intención, de información y de opinión.

Las principales variables de los cuestionarios fueron: a) Balance de la docencia virtual durante la etapa de confinamiento; b) Valoración de las actividades y los contenidos facilitados; y c) Análisis del rol de los docentes. Este instrumento fue validado por un panel de expertos $(n=6)$ en materia de comunicación y educación antes de ser implementado. Posteriormente se realizó una versión electrónica de la encuesta que fue enviada a tres facultades de comunicación, una por cada país. Tanto los usuarios como los formadores participantes fueron informados del estudio y su consentimiento fue solicitado para participar en el mismo. Con las encuestas se trazó un análisis motivacional exploratorio a partir de diferentes campos de trabajo: 
- Para los estudiantes: datos sobre edad, país y género; balance del periodo de docencia virtual durante el confinamiento y valoración de los contenidos, dinámicas de trabajo, habilidades del equipo docente e información recibida desde la universidad.

- Para los docentes: conocimiento de las características de la docencia virtual, dominio de las plataformas dialógicas y de las pautas de creación de contenidos para el ciberespacio.

En ambas encuestas, para la recogida de datos se utilizaron preguntas cerradas de escalas nominales excluyentes (en su mayoría a partir del uso de la escala Likert con un grado de respuesta, donde el 1 significa totalmente en desacuerdo y el 4 totalmente de acuerdo) y además diferentes preguntas abiertas. Se incluyeron preguntas cerradas dicotómicas para los temas más definidos sobre el tipo uso de internet y el conocimiento de aspectos vinculados con la navegación segura. Además, se consideró pertinente incorporar preguntas categorizadas (ofreciendo al encuestado una serie de listados de posibles opciones) y, de este modo, se combinaron preguntas de identificación, de intención, de información y de opinión. Las respuestas abiertas, que posibilitaron la escritura libre, permitieron conocer el grado de dominio de ciertos temas, así como la capacidad de explicación de los mismos por parte de los usuarios y de los formadores.

El análisis estadístico empleado en las encuestas fue descriptivo, basado en frecuencias y porcentajes, cuyas respuestas se equipararon en porcentajes con una intención comparativa. La consistencia interna del test presentó una alta fiabilidad con un Alpha de Cronbach de 0,943, confirmando medir el constructo de los múltiples factores intercorrelacionados. Según George y Mallery (2003), cuando el coeficiente de Alpha es >,90, podremos decir que la fiabilidad del instrumento es excelente y si es >,80 diremos que es bueno. El cuestionario se envió a través de la aplicación de Google Form.

\section{Resultados}

El paso de la presencialidad a la virtualidad ha sido considerado por la mayoría del alumnado como cambio negativo. Tanto en España (93\%), Ecuador (83,3\%) como en Italia (64,8\%), los estudiantes consideran que el cambio les ha perjudicado. La valoración sobre el cambio de la docencia presencial a la virtual ofrece resultados muy parejos entre los profesores que opinan que les ha beneficiado $(37,8 \%)$ y aquellos que argumentan que les ha perjudicado $(35,7 \%)$; mientras que un $26,5 \%$ valora dicho cambio como una modificación indiferente.

Este primer dato invita a una honda reflexión en el seno de las instituciones educativas. Los motivos de este prejuicio varían según los países (ver gráfico 1). En el caso de España, el alumnado alude a la peor calidad docente en un 39,5\% de los casos, a la mayor cantidad de lecturas y trabajos en un $27,9 \%$ y a la sensación de que aprenden menos en un 19\%. Un 13\% considera que el entorno virtual es menos estimulante. En el caso de Ecuador, no obstante, un 58,1\% identifica la perdida de motivación como principal elemento negativo, seguida de la sensación de aprender menos (en un $35,1 \%$ ). Las alusiones a una peor calidad docente, con un $4,1 \%$, y a la mayor cantidad de lecturas y trabajos, con un $2,7 \%$, son elementos muy puntuales. Finalmente, en el caso de Italia, la falta de estímulo ocupa la lista de prejuicio obtenidos en el cambio de modalidad de estudio con un 54,9\%, seguida de la mayor cantidad de trabajos y lecturas con un $28,6 \%$, y la peor calidad docente con un $12,1 \%$.

En este sentido, salvo la coincidencia entre los estudiantes ecuatorianos e italianos respecto a la falta de estímulos, se perciben importantes diferencias entre las tres realidades analizadas. Por su parte, entre el profesorado, de los que consideran que el cambio de la presencialidad a la virtualidad les ha perjudicado, un $38,7 \%$ señala que el principal problema ha sido el incremento del trabajo; mientras 
que un $32,3 \%$ considera que este nuevo escenario es menos estimulante. Además, un $24,7 \%$ cree que los alumnos aprenden menos en esta modalidad y un $4,3 \%$ señala que la calidad de la docencia empeora al pasar a la virtualidad.

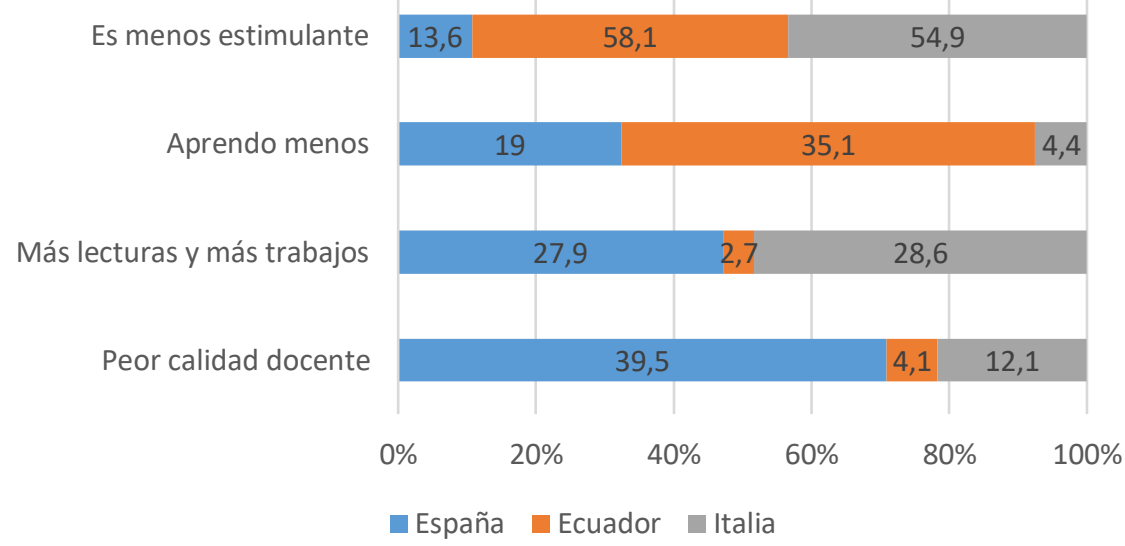

Gráfico 1: Motivos prejudicio para los estudiantes de la docencia virtual.

Fuente: elaboración propia.

Los elementos positivos que el alumnado señala del cambio de docencia (de la presencialidad a la virtualidad) destaca la posibilidad de gestionar su propio tiempo y la flexibilidad (ver gráfico 2). En el caso español, la flexibilidad (con un $30,6 \%$ ), la flexibilidad $(26,1 \%$ ) y la autonomía $(17,2 \%)$ son los elementos que el alumnado juzga más positivos en el cambio de escenario formativo. En Ecuador, la gestión del tiempo ocupa la primera posición $(34,4 \%)$, pero resulta llamativo que un $22,2 \%$ opina que no existe ningún elemento positivo en este cambio. La autonomía (con un 12,2\%) ocupa el tercer puesto. En el caso italiano, la gestión del tiempo es también el principal elemento positivo (con un 48,8\%), seguido de la flexibilidad $(23,2 \%)$ y la autonomía $(14,4 \%)$. Para los docentes, la posibilidad de gestionar el tiempo (40,3\%) es el principal aporte positivo. Seguidamente, un $23,5 \%$ valora la flexibilidad y un $15,8 \%$ hace referencia a la autonomía. Por su parte, la mayor cantidad de tareas es el elemento que juzgan como principal elemento negativo en un 39,3 de los casos.

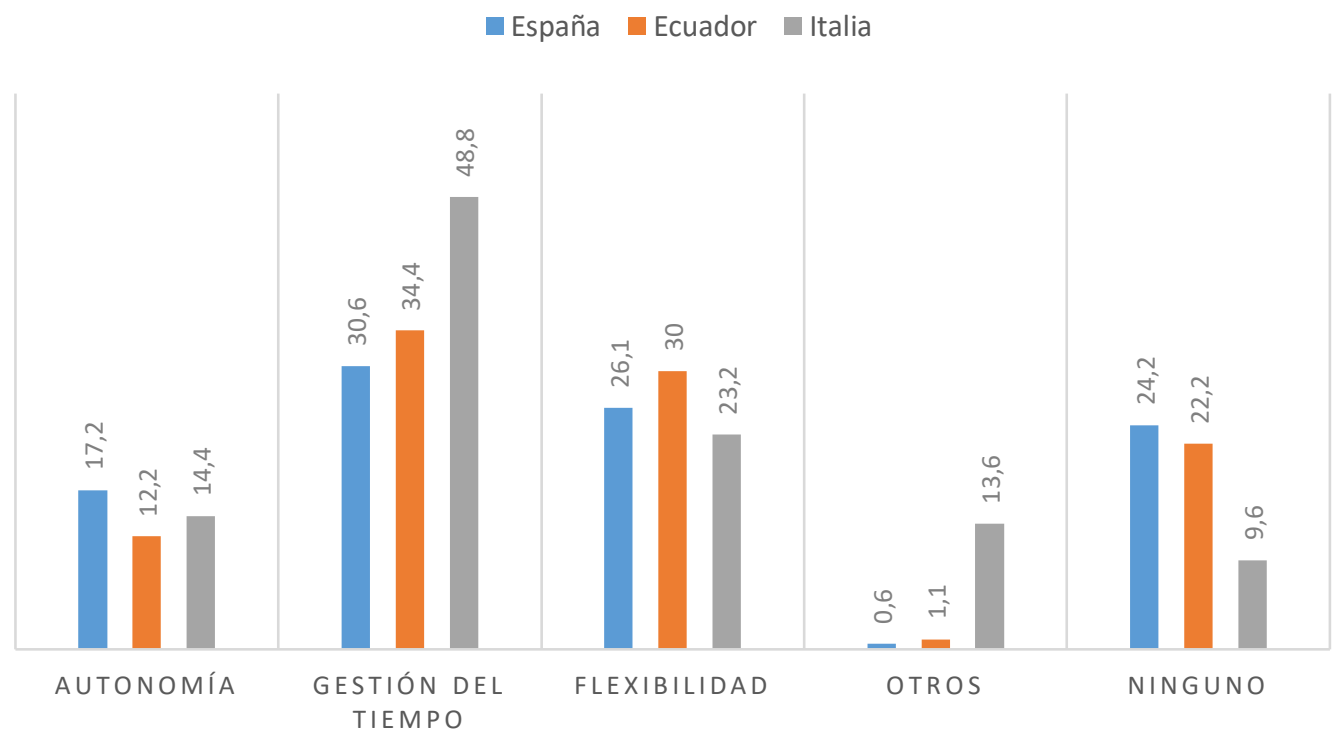

Gráfico 2: Elementos positivos del cambio de docencia (virtual por presencial).

Fuente: elaboración propia. 
El profesorado ha desempeñado un papel protagónico en la transición de la educación presencial a la virtual. Sin embargo, en muchos casos, este tránsito se ha realizado de forma precipitada. Resulta llamativo que tanto en Ecuador (76,7\%) como en Italia $(74,6 \%)$, el alumnado considera que sus respectivos docentes poseen las habilidades necesarias para diseñar la docencia virtual.

Únicamente, en el caso de España (con un 24,8\%) los estudiantes consideran que este requisito no existe. A lo anterior se unen las deficiencias que los estudiantes identifican en sus docentes para abordar el reto de la enseñanza virtual que ha impuesto la pandemia COVID-19 (ver gráfico 3). En el caso español, las principales deficiencias son, por este orden: la falta de adaptación de la docencia al nuevo escenario (83,2\%), el escaso dominio de los aspectos técnicos $(10,1 \%)$ y otros aspectos (6,7\%), como 'actividades injustificadas' o la sensación de realizar 'cosas inútiles' para su formación.

Por su parte, para el alumnado ecuatoriano, la falta de adaptación es el principal problema con un $73,9 \%$. Le siguen otros aspectos como la gran cantidad de dudas relacionadas con los encargos y ejercicios $(8,6 \%)$, el carácter inesperado del cambio de escenario (4,3\%), la falta de atención $(4,3 \%)$ o los "malos profesores" que los siguen siendo en la virtualidad $(4,3 \%)$. Finalmente, en el caso italiano, la carencia de dominio de los aspectos técnicos es el principal problema (con un 50\%), seguido de la falta de adaptación de la docencia $(31,6 \%)$ y de otros aspectos $(18,4 \%)$ como la improvisación permanente. Estos datos colisionan con las reflexiones de los docentes que, en un $91,8 \%$, de los casos, aseguran poseer las habilidades necesarias para afrontar la docencia virtual con solvencia.

- No dominan aspectos técnicos No adaptan docencia al nuevo escenario $\quad$ Otros

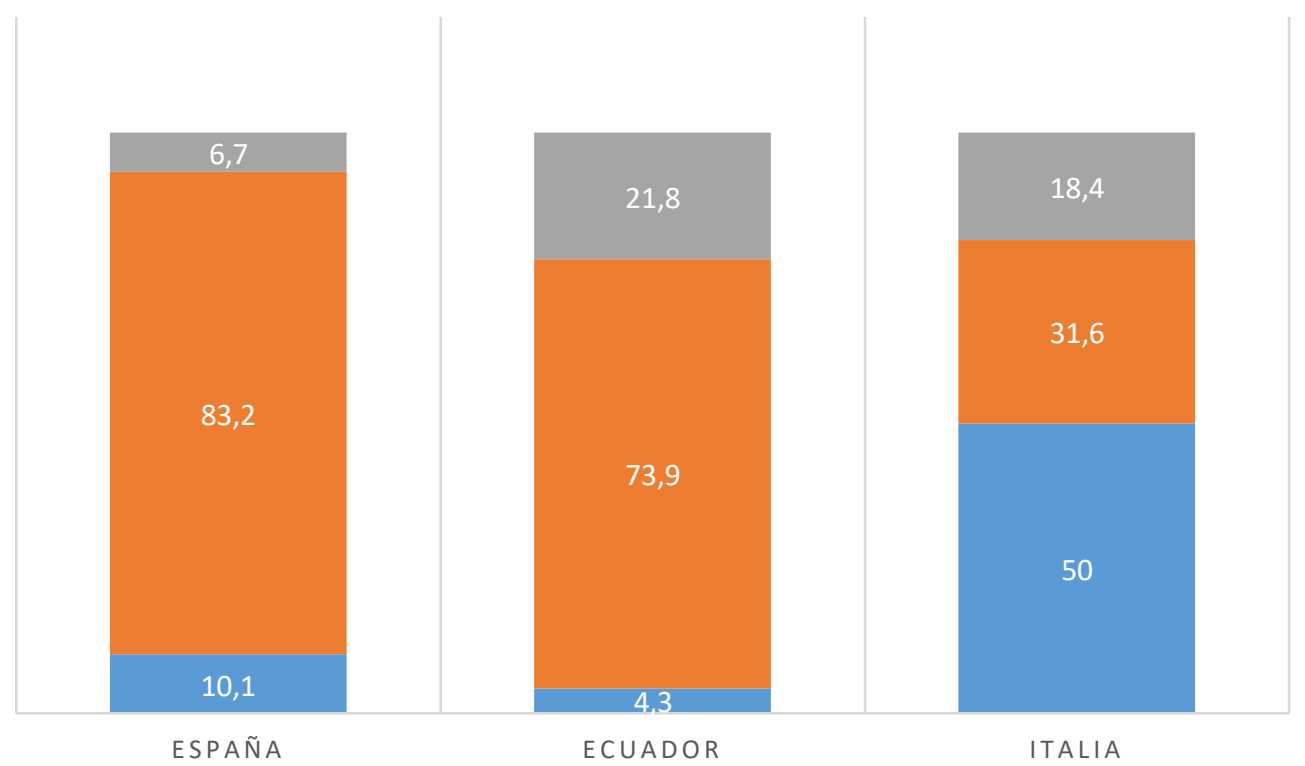

Gráfico 3: Principales deficiencias del profesorado para la enseñanza virtual

Fuente: elaboración propia.

Con relación al rol del profesorado, resulta igualmente importante destacar que tanto en Ecuador $(82,2 \%)$ como en Italia $(63,5 \%)$, el alumnado afirma que ha existido una coordinación entre los profesores de diferentes asignaturas, aspecto que no se da en el escenario español, donde solo un 14\% apunta que dicha coordinación ha existido. El alumnado de forma mayoritaria considera que posee las habilidades necesarias para seguir la docencia virtual. Este dato vierte resultados muy 
parejos en los tres países: España $(75,8 \%)$, Ecuador $(74,4 \%)$ e Italia $(73,6 \%)$. Estos porcentajes se acercan a la percepción que el profesorado posee de sus estudiantes. Un $75 \%$ de los docentes considera que estos poseen las habilidades necesarias para afrontar con éxito una docencia virtual.

No obstante, el alumnado se reconoce algunas deficiencias que se focalizan preferentemente la organización de su tiempo de estudio y en menor medida en el dominio de aspectos técnicos (ver gráfico 4). En el caso de España, un 71,8\% reconoce que le cuesta organizar su tiempo; mientras que un $12,8 \%$ reconoce no dominar los aspectos técnicos. Finalmente, un 15,4\% alude a otros aspectos como: la inexperiencia en gestionar este tipo de volúmenes de trabajo (2,6\%); la falta de consideración hacia los alumnos que estudian y trabajan $(2,6 \%)$; las elevadas exigencias técnicas por parte del docente $(2,6 \%)$; la falta de consideración hacia los aspectos vinculados con la salud mental $(2,63 \%)$ o la pereza o falta estímulo $(4,6 \%)$, entre otros. En el caso de Ecuador, por su parte, se observa que las principales deficiencias del alumnado se concentran en la ausencia de dominio de los aspectos técnicos (52\%), como sucede en Italia (44,1\%). Por otro lado, solo un $16 \%$ del alumnado ecuatoriano alud a dificultad para organizar el tiempo; mientras que un $32 \%$ se refiere a otros aspectos, como la falta en los hogares de equipamiento técnico o de la conectividad adecuada (20\%), el mayor número de distracciones $(4 \%)$ o la dificultad para realizar varias tareas al mismo tiempo (4\%). En el caso de Italia, un 41,2\% señala la dificultad para organizar su tiempo de estudio y un $14,7 \%$ alude a otros aspectos como las dificultades técnicas o la sensación de un trabajo desmesurado.

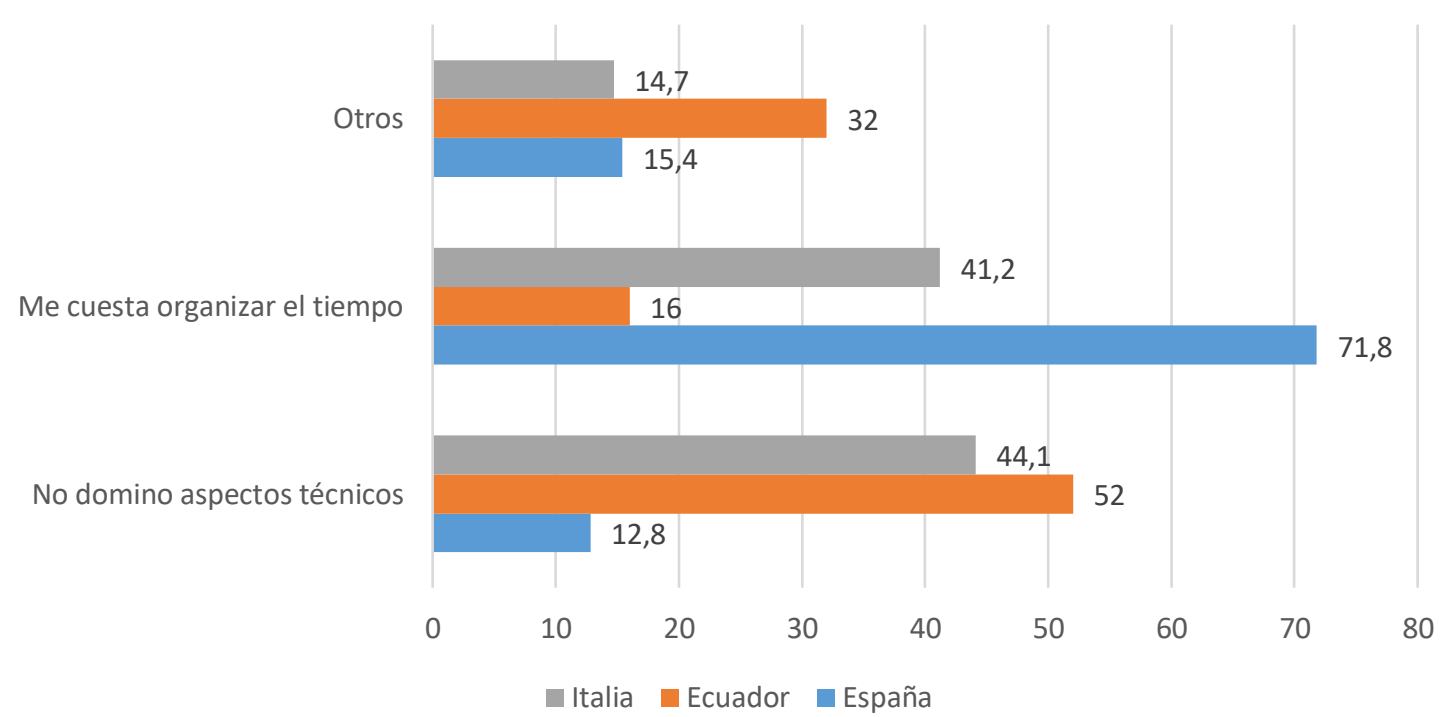

Gráfico 4. Principales deficiencias del alumnado para la enseñanza virtual.

Fuente: elaboración propia.

Las consecuencias negativas que el alumnado identifica en este cambio de docencia se centran en el caso español en el incremento de la carga de trabajo (para un 65,6\%) del alumnado, junto a la perdida de contacto presencial con los docentes $(19,1 \%)$ y con los compañeros $(12,1 \%)$. Estas valoraciones coinciden ampliamente con las reflexiones de los docentes que, en un 61,2\% de los casos, consideran que el peor elemento de la docencia virtual durante el confinamiento ha sido la perdida de contacto con el alumnado.

En el caso de Ecuador, el 43,3\% señala la pérdida del contacto con el profesorado como el principal agravio del cambio de escenario, seguida de la mayor carga de trabajos $(27,8 \%)$, la necesidad de ser más autónomos $(15,6 \%)$ y, finalmente, la perdida de contacto con los compañeros $(13,3 \%)$. En Italia, 
la principal consecuencia negativa se relaciona con la mayor carga de ejercicios y entregas $(39,7 \%)$, junto a la perdida de contacto con los docentes $(29,4 \%)$ y los compañeros $(27,8 \%)$. Solo un $3,1 \%$ señala la necesidad de ser más autónomo como una consecuencia negativa del cambio.

Resulta llamativo que en los tres países la imposibilidad del contacto presencial con el profesorado es considerado como uno de los elementos más negativos, ocupando ente todos los escenarios, la primera o segunda posición de importancia.

Las universidades han tenido que hacer frente de forma precipitada a una crisis que ha afectado contundentemente a la gestión universitaria. En este sentido, salvo en España (con un 43,9\%), en Ecuador (con un 61,1\%) e Italia (con un 52,8\%) la mayoría de los estudiantes reconocen haberse sentido bien informados por parte de sus respectivas universidades. España, con un 56,1\%, es el país donde se identifica un mayor número de alumnos que se reconocen desinformados.

En esta misma línea, la sensación de haber recibido informaciones e instrucciones contradictorias han sido numerosas en el caso el caso español e italiano. España (con un 65,6\%) e Italia (con un $72 \%$ ) presenta unos porcentajes elevados con relación a este aspecto. En el caso de Ecuador, un 41,1\% estaría dentro de este grupo; mientras que para un 58,9\% las indicaciones y la información recibida no ha sido, en ningún momento, contradictoria. Finalmente, el alumnado otorga una calificación general cercana al aprobado en los tres países con algunas particularidades (ver gráfico 5). En España, el alumnado confiere un suspenso con un $49 \%$ frente al $42 \%$ de quienes le otorgan un aprobado. En el caso ecuatoriano, la calificación con mayor protagonismo es el notable $(44,4 \%)$, frente al aprobado mayoritario de Italia (con un 63,5\%).

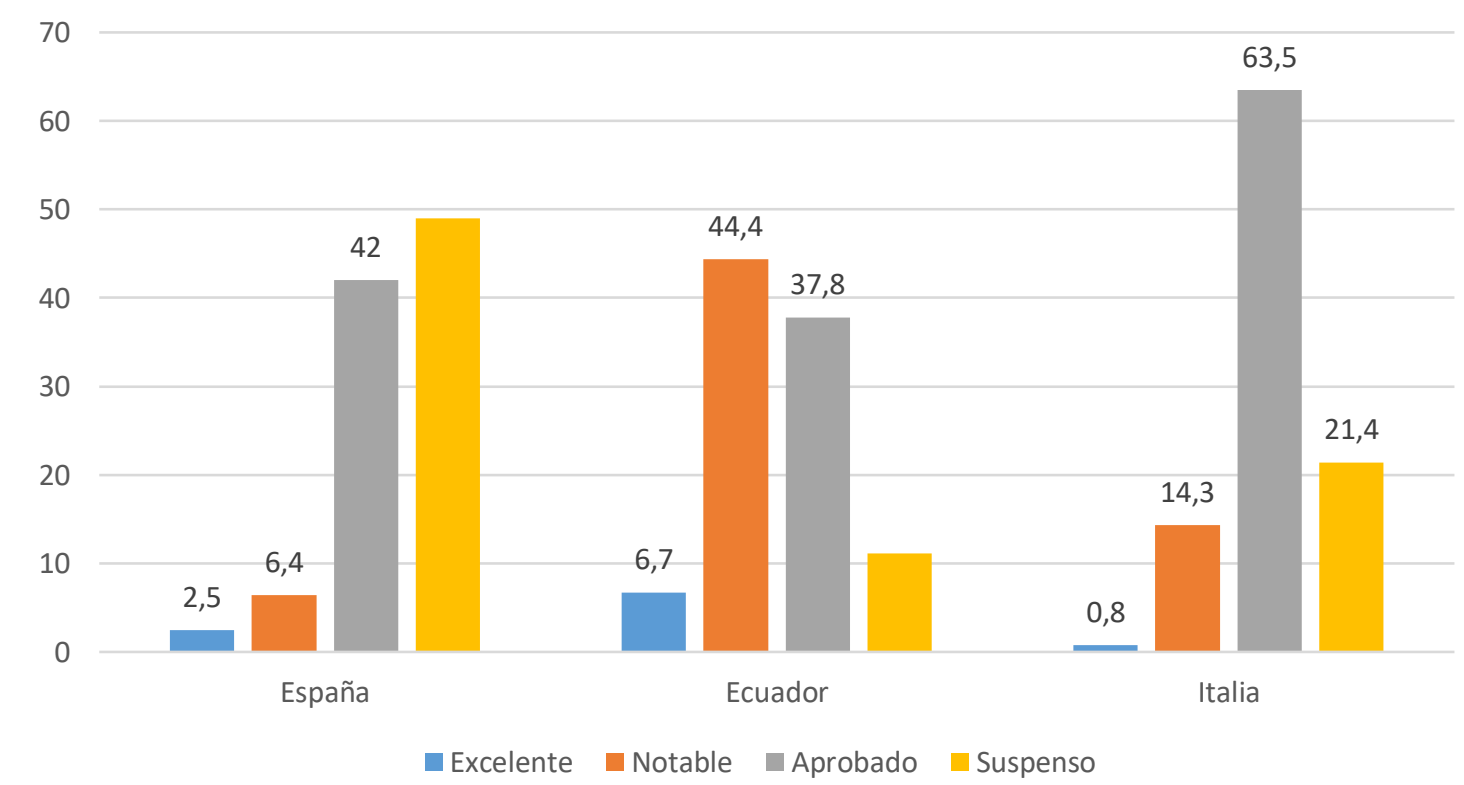

Gráfico 5: ¿Qué nota le pondrías a tu universidad en la forma de gestionar el impacto de esta crisis en la docencia?

Fuente: elaboración propia.

Frente a estos resultados, el 76,5\% del profesorado reconoce haberse sentido bien informado de las diferentes situaciones, decisiones y novedades informativas ligadas a la pandemia por parte de sus universidades. En esta línea, la mayoría de los docentes considera que, si hubiera que calificar a sus universidades, estas han logrado aprobar, aunque las calificaciones varían: para un 37,2\% sería de 
aprobado; para un $34,7 \%$, de notable; y para un 18,9\% de excelente. Únicamente, un 9,2\% suspende a sus respectivas universidades.

\section{Discusión}

Los resultados de esta investigación concuerdan con los estudios de Mishra y Koehler (2006) y Koehler y Mishra (2008) para quienes el docente, en el escenario de la teleformación, debe mostrar competencias alrededor de tres grandes componentes: disciplinar, pedagógico y tecnológico, cuyas dimensiones son: 1) conocimiento sobre el contenido de la materia; 2) conocimiento de los procesos y las prácticas del método de enseñanza; 3) conocimiento pedagógico de contenido; conocimiento de las TIC estándar que se utilizan en la enseñanza; 4) conocimiento de la utilización de las TIC en los procesos de enseñanza; y 5) conocimiento tecnológico, pedagógico y de contenido.

De igual manera, la percepción del profesorado de Italia, España y Ecuador rescata la teoría ofrecida por Kirschner y Davis (2003), quienes formularon seis grandes bloques para las acciones formativas que se desarrollen en TIC, las cuales son: 1) competencia para el uso personal de las TIC; 2) competencia para hacer uso de las TIC como herramientas de la mente, 3) dominio de una serie de paradigmas educativos que hacen uso de las TIC; 4) competencia para hacer uso de las TIC como una herramienta para la enseñanza; 5) competencia en una amplia gama de paradigmas de evaluación que hacen uso de las TIC; 6) comprensión de la dimensión política de la utilización de las TIC para la enseñanza y el aprendizaje, propuestas que han sido acogidas incluso por entidades internacionales como el Departamento de Educación de Australia (Pearson, 2003).

La calidad de la comunicación en los cursos online, es una de las variables más señaladas en la literatura como precedente de la satisfacción del alumnado (Wang y Chiu, 2011), indicador que también se repite en esta investigación. Para los estudiantes italianos, ecuatorianos y españoles, la comunicación, impulsada por el profesor, es clave en la apreciación de calidad de los cursos online (Emeyano y Voronina, 2014). Esta relación ha de frecuente para alcanzar un intercambio de conocimientos y un respeto mutuo que permita la satisfacción de todos los actores educativos (Margalina, De Pablos et al., 2014). A más de ello, la proximidad social en estos entornos provoca una valoración positiva por parte del estudiante (Kim et al., 2011).

Los estudiantes de Italia, España y Ecuador enfatizan en que los entornos virtuales de aprendizaje deben dotar de libertad al alumnado y focalizar su interés en este, propiciando su autonomía y formación continua, criterio que también se reivindica en los estudios de Salinas (2002, 2004). Para este autor, una exitosa experiencia educativa online depende de la adecuación de los recursos humanos y materiales, la adquisición de diferentes comportamientos y la asimilación de esta nueva cultura.

Otro factor que se destaca, desde la visión de los jóvenes universitarios de los tres países, es la importancia del trabajo colaborativo, que se enuncia en la teoría de Bolívar (2006), puesto que son necesarios los vínculos, síncronos y asíncronos, entre los diferentes agentes educativos. Para su cumplimiento idóneo, hay que mejorar los procesos de tutorías y canales de comunicación en los entornos virtuales de aprendizaje, con el fin de construir un proyecto pedagógico con fines comunes que apunten al desarrollo integral del discente (García, Gomáriz, Hernández y Parra, 2010).

Para los estudiantes encuestados, la función de los docentes, en los entornos virtuales, no ha de limitarse a la mera transmisión de conocimientos especializados (Prieto, 2008), sino que estos deben adaptarse a las transformaciones de la sociedad en el escenario de la 'nueva normalidad' y plantear 
modelos activos y lúdicos, a través de estrategias metodológicas interactivas e inmersivas (Robizo y Cózar, 2015).

Desde la perspectiva del alumnado italiano, ecuatoriano y español, el docente tiene que ser capaz de innovar, reflexionar y transformar sus propuestas didácticas para responder a las demandas sociales que vive el mundo en medio de una crisis sanitaria, a la vez que se alcancen los objetivos curriculares propuestos al inicio del curso (Paredes, 2010).

Por otro lado, los docentes encuestados reconocen que es necesario la promoción del pensamiento crítico y reflexivo vinculado a la gestión estratégica de las TIC (Bonilla del Río y Aguaded, 2018), con el fin de fomentar prácticas que superen el ejercicio de las habilidades instrumentales y busquen un uso efectivo de los recursos tecnológicos (Arrieta y Montes, 2011).

La implementación de las TIC en los programas de Educación Superior depende de docentes digitalmente competentes (Agreda, Hinojo y Sola, 2016; Gisbert, Espuny y González, 2011; Rangel, 2015; Valdivieso y Gonzáles, 2016), una valoración presente en esta investigación tanto para los estudiantes implicados como para los docentes encuestados.

Los estudiantes de Italia, España y Ecuador conciben el apoyo y la orientación de sus docentes como un factor clave de éxito educativo que asegura el aprovechamiento integral de las TIC (Salinas, 2004). Ello garantiza la calidad de la formación on-line, siempre que se den cumplimiento a los aspectos funcionales (interés, eficacia, versatilidad) relacionados con el entorno (sencillez, claridad, originalidad), relacionados con el modelo pedagógico (plan docente, adecuación, flexibilidad, evaluación) y relacionados con los servicios complementarios (información, noticias, agenda) (Santoveña, 2010) de los Entornos Virtuales de Aprendizajes (EVA) de sus respectivas universidades.

Investigaciones previas demuestran que los profesores expresan actitudes positivas frente a la utilización e incorporación de las TIC en los procesos formativos (Jimoyiannis y Komis, 2007; Banas, 2010; Álvarez et al., 2011), siendo estas empleadas en mayor medida en actividades de investigación que en docencia (Maroto, 2007; Mcvee et al., 2008), un dato que sale a relucir en este estudio. Por mencionar un caso, los estudiantes ecuatorianos opinan que a sus docentes les falta mayor incorporación de las tecnologías educativas en sus dinámicas de enseñanza, puesto que en los entornos virtuales vuelven a emplear el recurso de la clase magistral. Los jóvenes universitarios buscan que el uso de la tecnología ponga como centro del aprendizaje al alumnado (Bartolomé, 2004); sin embargo, esto no ocurre al $100 \%$. Para que se dé una experiencia educativa satisfactoria en el ciberespacio, los estudiantes deben poseer competencias básicas que les permitan trabajar con los entornos educativos basados en la tecnología inmersiva, un dato al que apuntan los docentes encuestados y que es recomendación de Centeno Moreno y Cubo Delgado (2013).

Los hallazgos de esta investigación tienen como fundamento la perspectiva de Llorente, (2008) y Barroso Osuna et al. (2012), puesto que los docentes encuestados, particularmente ecuatorianos, consideran que las capacitaciones institucionales tienen que superar el carácter instrumental de las TIC e incluirse dimensiones complementarias basadas en: semiológica-estética, currículo, pragmática, psicología educativa, diseño gráfico, evaluación, organización del tiempo, así como habilidades investigadoras.

Además, estos autores recomiendan dar valor a la práctica, a los contextos naturales de enseñanza y a la coproducción de materiales entre profesores y expertos. También contemplar el estudio de problemas reales y situar al docente en el uso de estrategias de formación más amplias. 


\section{Conclusiones}

Esta investigación ha permitido analizar y, al mismo tiempo, contrastar los puntos de vista del alumnado y del profesorado de tres países donde el impacto de la COVID-19 ha sido alarmante. En el caso de los estudiantes de pregrado de las áreas de Comunicación, Periodismo y Educación de la Universidad de Torino (Italia), Universidad Autónoma de Barcelona (España) y Universidad Técnica de Machala (Ecuador) se observa una valoración negativa sobre el paso a la virtualidad de la Educación Superior.

Por parte del claustro docente la valoración es menos crítica y denota una cierta indiferencia frente a este cambio. Sin embargo, no es posible señalar que el alumnado identifique los mismos elementos negativos. Los aspectos coyunturales, o incluso el tipo de estudios cursados, son factores que podrían tener incidencia en la mayor o menor satisfacción del alumnado frente a este cambio de régimen de estudio.

El escenario de la 'nueva normalidad' ha incrementado la importancia de la gestión del tiempo, la autonomía y la flexibilidad en la formación de los futuros profesionales. Este aspecto resulta crucial en la medida en que invita a repensar y, por ende, redefinir, el conjunto de competencias, contenidos y logros de aprendizaje de los programas formativos de los distintos grados.

De acuerdo a los resultados de la investigación, la virtualidad se asocia, de forma recurrente, con un incremento de la carga lectiva, aspecto que denota la necesidad de revisar los procesos formativos y establecer indicadores y parámetros eficaces en este ámbito, con el objetivo de no saturar al alumnado de actividades extracurriculares que pueden ocasionar la deserción del mismo.

Por otro lado, la visión que el alumnado posee de sus docentes en el escenario virtual arroja importantes inquietudes pues ese cambio ha contribuido a impactar negativamente en la visión que estos proyectan como formadores. Desde la percepción del profesorado, se valora la capacidad técnica de sus estudiantes, pero consideran que los alumnos necesitan seguir reforzando competencias relativas al uso crítico y reflexivo de las tecnologías. Más allá de habilidades instrumentales, se demanda la construcción de una ciudadanía digital activa y empoderada.

Finalmente, la pandemia del coronavirus ha contribuido a avivar el peso de las diferencias contextuales, que abarcan desde problemas de conectividad a la calidad de las prestaciones tecnológicas de los discentes de Ecuador, España e Italia, advirtiendo diferentes escenarios de desigualdad educativa que podrían generar futuras crisis sociales en los tres países analizados. Al término del estudio, la pérdida de la presencialidad se erige como el principal elemento negativo tanto para alumnos como para profesores, aspecto decisivo en el diseño de futuras estrategias educativas virtuales en épocas de confinamiento.

\section{Bibliografía}

Agreda, M., Hinojo, M. A. y Sola, J. M. (2016). Diseño y validación de un instrumento para evaluar la competencia digital de los docentes en la educación superior española. Píxel-Bit, (49), 39-56. http://dx.doi.org/10.12795/pixelbit.2016.i49.03

Aguaded, J. I. y Díaz, M. R. (2009). Presencia virtual de las universidades andaluzas. Revista @tic,

3, 18-28. Recuperado de http://hdl.handle.net/10272/6312 
Álvarez, S. y otros (2011). Actitudes de los profesores ante la información de las TIC en la práctica docente. Estudio de un grupo de la Universidad de Valladolid. Edutec. Revista Electrónica De Tecnología Educativa, 35, a160. https://doi.org/10.21556/edutec.2011.35.416

Aquino, E. M. L., Silveira, I. H., Pescarini, J. M., Aquino, R., Souza-Filho, J. A. de Rocha, A. S., et al. (2020). Medidas de distanciamento social no controle da pandemia de COVID-e19: potenciais impactos e desafios no Brasil. Ciência \& Saúde Coletiva, 25(1), 2423-2446. https://dx.doi.org/10.1590/1413-81232020256.1.10502020

Area, M. (2010). ¿Por qué formar en competencias informacionales y digitales en la educación superior? Revista de Universidad y Sociedad del Conocimiento, 7(2), 2-5. http://dx.doi.org/10.7238/rusc.v7i2.976

Arrieta, A. y Montes, V. D. (2011). Alfabetización digital: uso de las TIC más allá de una formación instrumental y una buena infraestructura. Revista Colombiana de Ciencia Animal, 3(1), 180-197. https://doi.org/10.24188/recia.v3.n1.2011.360

Ballestero, C. y otros. (2010). Usos del e-learning en las universidades andaluzas. Estado de la situación y análisis de buenas prácticas. Pixel-Bit, 37, 7-18. Recuperado de https://recyt.fecyt.es/index.php/pixel/article/view/61395

Banas, J. (2010). Teachers' Attitudes toward Technology. Considerations. Community \& Junior College Libraries, 16(2), 114-127. https://doi.org/10.1080/02763911003707552

Bartolomé, A.R. (2004). Blended learning. Conceptos básicos. Pixel-Bit, 23, 7-20. Recuperado de https://www.redalyc.org/pdf/368/36802301.pdf

Bekerman, U. y Rondanini, A. (2020). El acceso a internet como garantía del derecho a la educación. Diario DPI Suplemento Salud, 58, 1-7. http://dx.doi.org/10.2139/ssrn.3576719

Beltrán, J., Venegas, M., Villar-Aguilés, A., Andrés-Cabello, S., Jareño-Ruiz, D. y de GraciaSoriano, P. (2020). Educar en época de confinamiento: La tarea de renovar un mundo común. Revista de Sociología de la Educación, $13(2), \quad 92-104$. http://dx.doi.org/10.7203/RASE.13.2.17187

Berelson, B. (1971). Content analysis in communication research. Harper.

Bolívar, A. (2006). Familia y escuela: dos mundos llamados a trabajar en común. Revista de educación, 339, 119-146. Recuperado de http://www.educacionyfp.gob.es/dam/jcr:3edbf2a89420-43fb-9b47-fb7044cc74de/re33908-pdf.pdf

Bonilla-del-Río, M. y Aguaded, I. (2018). La escuela en la era digital: smartphones, apps y programación en Educación Primaria y su repercusión en la competencia mediática del alumnado. Píxel-Bit, 53, 151-163. https://doi.org/10.12795/pixelbit.2018.i53.10

Bozkurt, A.O., Yilmazel, E., Ucar, G., Sezgin, K., Sen-Ersoy, G.C., Dincer, A. \& Aydin (2015). Trends in distance education research: A content analysis of journals 2009-2013. The International Review of Research in Open and Distributed Learning, 16(1), 330-363. https://doi.org/10.19173/irrodl.v16i1.1953

Bulut Z. A.; Dogan O. (2017). The ABCD typology: Profile and motivations of Turkish social network sites users. Computers in Human Behavior, 67, 73-83. https://doi.org/10.1016/j.chb.2016.10.021 
Cabero Almenara, J. (2014). Formación del profesorado universitario en TIC. Aplicación del método Delphi para la selección de los contenidos formativos. Educación XX1, 17(1), 1-32. https://doi.org/10.5944/educxx1.17.1.10707

Cáceres-Muñoz, J., Jiménez Hernández, A. S. y Martín-Sánchez, M. (2020). Cierre de Escuelas y Desigualdad Socioeducativa en Tiempos del Covid-19. Una Investigación Exploratoria en Clave Internacional. Revista Internacional de Educación para la Justicia Social, 9(3). https://doi.org/10.15366/riejs2020.9.3.011

Cao, W., Fang, Z., Hou, G., Han, M., Xu, X., Dong, J. \& Zheng, J. (2020). The psychological impactof the Covid-19 epidemic on college students in China. Psychiatry Research, 287(112934), 1-5. https://doi.org/10.1016/j.psychres.2020.112934

Carla Silva, T., Ramos Oliveira, E. y Montanari, R. (2020). Dificultades de la educación remota en las escuelas rurales del norte de Minas Gerais durante la pandemia de Covid-19. Research, Society and Development, 9(8). http://dx.doi.org/10.33448/rsd-v9i8.6053

Centeno Moreno, G. y Cubo Delgado, S. (2013). Evaluación de la competencia digital y las actitudes hacia las TIC del alumnado universitario. Revista de Investigación Educativa, 31(2), 517-536. https://doi.org/10.6018/rie.31.2.169271

Cervantes Holguín, E. y Gutiérrez Sandoval, P. R. (2020). Resistir la Covid-19. Intersecciones en la Educación de Ciudad Juárez, México. Revista Internacional de Educación para la Justicia Social, 9(3). https://doi.org/10.15366/riejs2020.9.3.001

Chen, Ch. (2008). Why Do Teachers Not Practice What They Believe Regarding Technology Integration? Journal of Educational Research, 102(1), 65-75. https://doi.org/10.3200/JOER.102.1.65-75

Colás, P., González, T., De Pablos, J. (2013). Juventud y redes sociales: Motivaciones y usos preferentes. Comunicar, 40, 15-23. https://doi.org/10.3916/C40-2013-02-01

Corrêa Filho, H. R., \& Segall-Corrêa, A. M. (2020). Lockdown ou vigilância participativa em saúde? Lições da Covid-19. Saúde em Debate, 44(124), 5-10. https://doi.org/10.1590/01031104202012400

Davies, R., Howell, S. \& Petrie, J. (2010). A review of trends in distance education scholarship at research universities in North America, 1998-2007. The International Review of Research in Open and Distance Learning, 11(3), 42-56. https://doi.org/10.19173/irrodl.v11i3.876

Del Rincón, D., Arnal, J., Latorre, A., Sans, A. (1995). Técnicas de Investigación en Ciencias Sociales. Dykinson.

Emelyanova, N. and Voronina, E. (2014). Introducing a learning management system at a russian university: students' and teachers' perceptions. The International Review Research in Open and Distance Learning, 15(1), 272-289. https://doi.org/10.19173/irrodl.v15i1.1701

Feyen, J. (2020). ¿Logrará la COVID-19 acelerar la transición del aprendizaje pasivo a la educación activa?. Maskana, 11(1), 1-4. https://doi.org/10.18537/mskn.11.01.00

Fontana-Hernández, A., Herrera-Sibaja, S., Leiva-Durán, B., \& Montero-Cascante, J. (2020). El Proyecto UNA Educación de Calidad en el contexto de la COVID 19. Revista Electrónica $\begin{array}{lrl}\text { Educare, } & 24 . & \text { Recuperado } \\ \text { https://www.revistas.una.ac.cr/index.php/EDUCARE/article/view/14225 }\end{array}$ 
Freire, J. (2009). Monográfico cultura digital y prácticas creativas en educación. RUSC, Revista de Universidad y Sociedad del Conocimiento, 6(1). http://dx.doi.org/10.7238/rusc.v6i1.23

García, M.P., Gomáriz, M.Á., Hernández, M.Á. y Parra, J. (2010). La comunicación entre la familia y el centro educativo, desde la percepción de los padres y madres de los alumnos. Educatio siglo XXI, 28(1), 157- 187. https://revistas.um.es/educatio/article/view/109771

Garrote Rojas, D., Jiménez-Fernández, S. y Serna Rodríguez, R. (2018). Gestión del tiempo y uso de las TIC en estudiantes universitarios. Píxel-Bit, 53, 109-121. https://doi.org/10.12795/pixelbit.2018.i53.07

George, D., Mallery, P. (2003). SPSS for Windows step by step: A simple guide and reference. 11.0 update. Allyn \& Bacon.

Gisbert, M., Espuny, C. y González, J. (2011). INCOTIC. Una herramienta para la @utoevaluación diagnóstica de la competencia digital en la universidad. Profesorado, 15(1), 75-90. Recuperado de https://recyt.fecyt.es/index.php/profesorado/article/view/42011

Goktas, Y. et al. (2008). A review of ICT related courses in pre-service teacher education programs. Asia Pacific Education Review, 9(2), 168-179. Recuperado de https://files.eric.ed.gov/fulltext/EJ811128.pdf

González-Calvo, G., Barba-Martín, R.A., Bores-García, D. y Gallego-Lema, V. (2020). Aprendiendo a Ser Docente Sin Estar en las Aulas. La COVID-19 Como Amenaza al Desarrollo Profesional del Futuro Profesorado. International and Multidisciplinary Journal of Social Sciences, 2(9), 152177. http://doi.org/10.17583/rimcis.2020.5783

Harnad,S. (1991). Post-Guttemberg Galaxy: The Fourth Revolution in the Means of production of Knowledge. The Public-Access Computer System Review, 2(1), 39-53. Recuperado de https://eprints.soton.ac.uk/253376/1/harnad91.postgutenberg.html

Hammond, M. (2009). What happens as student teachers who made very good use of ICT during preservice training enter their first year of teaching? Teacher Development, 13(2), 93-106. https://doi.org/10.1080/13664530903043939

Herrera, L. A., Tusa, F. y Maza-Córdova, J. (2019). La universidad ecuatoriana como entorno inclusivo. El derecho a una educación integral. Revista Espacios, 40(8). Recuperado de http://www.revistaespacios.com/a19v40n08/19400809.html

Holsti, O. (1969). Content analysis for the social sciences and the humanities. Addison-Wesley.

Jimoyiannis, A. and Komis, V. (2007). Examining teachers' beliefs about ICT in education. implications of a teacher preparation programme. Teacher Development, 11(2), 149-173. https://doi.org/10.1080/13664530701414779

Kim, J., Kwon, Y. \& Cho, D. (2011). Investigating factors that influence social presence and learning outcomes in distance higher education. Computers \& Education, 57(2), 1512-1520. https://doi.org/10.1016/j.compedu.2011.02.005

Krippendorff, K. (1990). Metodología del análisis de contenido. Barcelona: Paidós.

Kirschner, P. and Davis, N. (2003). Pedagogic benchmarks for information and communications technology in teacher education. Technology, Pedagogy and Education, 12(1), 125-147. https://doi.org/10.1080/14759390300200149 
Koehler, J. and Mishra, P. (2008). Introducing Technological Pedagogical Knowledge. En The Handbook of Technological Pedagogical Content Knowledge for Educators. Taylor \& Francis Group.

Llorente, M.C. (2008). Aspectos fundamentales de la formación del profesorado en TIC. Pixel-Bit, 31, 121-130. Recuperado de https://recyt.fecyt.es/index.php/pixel/article/view/61291

López Carmona, L., López Carmona, B. y Prieto Jiménez, E. (2018). Tendencias innovadoras en la formación on-line. La oferta web de postgrados e-learning y blendedlearning en España. Píxel-Bit, 53, 1-15. https://doi.org/10.12795/pixelbit.2018.i53.06

Margalina, V.M.; De Pablos, C. et al. (2014). The role of relational coordination in final teacher satisfaction in e-learning. Procedia Technology, $16, \quad 365-375$. https://doi.org/10.1016/j.protcy.2014.10.102

Maroto, A. (2007). El uso de las nuevas tecnologías en el profesorado universitario. Pixel-Bit, 39, 211-223. Recuperado de https://recyt.fecyt.es/index.php/pixel/article/view/61303

Martínez-Caro, E. (2008). E-Learning: Un análisis desde el punto de vista del alumno. Revista Iberoamericana de Educación a Distancia, 11(2), 151-168. https://doi.org/10.5944/ried.2.11.948

Mcvee, M. et al. (2008). Teachers and teacher educators learning from new literacies and new technologies. Teaching Education, 19(3), 197-210. https://doi.org/10.1080/10476210802250216

Millán, T., Heresi, C., Díaz, F., Weisstaub, G. y Catalán, N. A. V. (2020). La pandemia COVID-19 como oportunidad de reflexión en Educación en Ciencias de la Salud. Revista Chilena de Pediatría, 91(4). http://dx.doi.org/10.32641/rchped.v91i4.2784

Mishra, P. and Koehler, J. (2006). Technological Pedagogical Content Knowledge. A new framework for teacher knowledge. Teachers College Record, 108(6), 1017-1054. https://www.doi.org/10.1111/j.1467-9620.2006.00684.x

Monasterio, D. y Briceño, M. (2020). Educación mediada por las Tecnologías: Un desafío ante la coyuntura del Covid-19. Observador del Conocimiento, 5(1), 136-148. Recuperado de http://www.oncti.gob.ve/ojs/index.php/rev_ODC/article/view/132

Morales, J. (2020). Oportunidad o Crisis Educativa: Reflexiones desde la Psicología para Enfrentar los Procesos de Enseñanza-Aprendizaje en Tiempos de Covid-19. Revista Internacional de Educación para la Justicia Social, 9(3). Recuperado de https://revistas.uam.es/riejs/article/view/12228

Olivares Carmona, K., Angulo Armenta, D., Prieto Méndez, D. y Torres Gastelú, D. (2018). EDUCATIC: implementación de una estrategia tecnoeducativa para la formación de la $\begin{array}{llll}\text { competencia digital } & \text { universitaria. } & \text { Píx-Bit, } & \text { 53, }\end{array}$ https://doi.org/10.12795/pixelbit.2018.i53.02

Pearson, J. (2003). Information and Communications Technologies and Teacher Education in Australia. Technology, Pedagogy and Education, 12(1), 39-58. https://doi.org/10.1080/14759390300200145

Pedró, F. (2020). COVID-19 y educación superior en América Latina y el Caribe: efectos, impactos y recomendaciones políticas. Análisis Carolina, (36). Recuperado de https://www.fundacioncarolina.es/wp-content/uploads/2020/06/AC-36.-2020.pdf 
Pérez-Escoda, A. Castro-Zubizarreta, A. y Fandos-Igado, M. (2016). La competencia digital de la Generación Z: claves para su introducción curricular en la Educación Primaria. Comunicar, 24(49). https://doi.org/10.3916/C49-2016-07

Prieto, E. (2008). El papel del profesorado en la actualidad. Su función docente y social. Foro de educación, 6(10), 325-345. $\quad$ Recuperado de https://dialnet.unirioja.es/servlet/articulo?codigo $=2907073$

Quevedo-Ramírez, E. J. (2020). Educación a distancia. Política educativa y escenario tecnosociológico venezolano en tiempos de Covid-19. Revista EDUCARE-UPEL-IPB-Segunda Nueva Etapa 2.0, 24(2), 308-322. https://doi.org/10.46498/reduipb.v24i2.1332

Rangel, A. (2015). Competencias docentes digitales: propuesta de un perfil. Píxel-Bit, 46, 235-248. https://doi.org/10.12795/pixelbit.2015.i46.15

Ríos Campos, C. (2020). COVID-19 y Educación Superior Universitaria Pública del Perú. Revista Clake Education, 1(02), 1-1. $\quad$ Recuperado de http://revistaclakeeducation.com/ojs/index.php/Multidisciplinaria/article/view/16

Robizo, M.J. y Cózar, R. (2015). Usos y competencias en TIC en los futuros maestros de educación infantil y primaria: hacia una alfabetización real para docentes. Pixel-Bit, 47, 23-39. https://doi.org/10.12795/pixelbit.2015.i47.02

Barroso Osuna, J. y otros. (2012). La formación del profesorado en TIC, visión enfocada en la enseñanza y el aprendizaje. Global, 48, 48-55. Recuperado de http://revista.global/la-formaciondel-profesorado-en-tic-vision-enfocada-en-la-ensenanza-y-el-aprendizaje/

Salinas, J. (2002). Modelos flexibles como respuesta de las universidades a la sociedad de la información. Acción Pedagógica, 11(1), 4-13. Recuperado de https://dialnet.unirioja.es/servlet/articulo?codigo=2973024

Salinas, J. (2004). Innovación docente y uso de las TIC en la enseñanza universitaria. Revista de Universidad y Sociedad del Conocimiento, 1(1), 1-16. http://dx.doi.org/10.7238/rusc.v1i1.228

Santoveña, S. (2010). Cuestionario de evaluación de la calidad de los cursos virtuales de la UNED. RED, Revista de Educación a Distancia, 25, 1-22. Recuperado de https://revistas.um.es/red/article/view/125311

Tarcízio, I. (2020). Obstáculos do ensino à distância na rede pública durante a pandemia de Covid19. Socialismo Criativo. Disponible en: https://bit.ly/3ifJkl4

Tenente, L. (2020). Sem internet, merenda e lugar para estudar: veja obstáculos do ensino à distância na rede pública durante a pandemia de Covid-19. Globo. Disponible en: https://glo.bo/3bK9n16

Tejedor, S., Bugs, R. y Luque, S. (2018). Los estudiantes de Comunicación en las redes sociales: estudio comparativo entre Brasil, Colombia y España. Transinformação, 30(2), 267-276. https://doi.org/10.1590/2318-08892018000200010

Teo, T. et al. (2008). Beliefs about teaching and uses of technology among preservice teaching. AsiaPacific Journal of Teacher Education, $36(2), \quad 163-174$. https://doi.org/10.1080/13598660801971641 
Valdivieso, T. S. y Gonzáles, M. A. (2016). Competencia digital docente: ¿Dónde estamos? Perfil del docente de educación primaria y secundaria. El caso de Ecuador. Píxel-Bit, 49, 57-73. Recuperado de https://recyt.fecyt.es/index.php/pixel/article/view/61714

Valdés, A. y otros. (2010). Necesidades de capacitación de docentes de educación básica en el uso de las TIC. Pixel-Bit, 39, 211-223. Recuperado de https://recyt.fecyt.es/index.php/pixel/article/view/61460

Valerio, C. y Paredes, J. (2008). Evaluación del uso y manejo de las tecnologías de información y comunicación en los docentes universitarios. Un caso mexicano. Revista Latinoamericana de Tecnología Educativa, 7(1), 13-32. Recuperado de https://relatec.unex.es/article/view/391

Vera, J. A., Torres, L. E. y Martínez, E. E. (2014). Evaluación de competencias básicas en TIC en docentes de educación superior. Pixel-Bit, 44 44, 143-155. https://doi.org/10.12795/pixelbit.2014.i44.10

Vilches, L. (2011). La investigación en comunicación. Métodos y técnicas en la era digital. Gedisa.

Villafuerte, J., Bello, J., Cevallos, Y. y Bermello J. (2020). Rol de los docentes ante la crisis del Covid-19, una mirada desde el enfoque humano. REFCalE: Revista Electrónica Formación y Calidad Educativa, 8(1), 134-150. Recuperado de https://refcale.uleam.edu.ec/index.php/refcale/article/view/3214

Wang, H.C. \& Chiu, Y.F. (2011). Assessing e-learning 2.0 system success. Computers \& Education, 57(2), 1790-1800. https://doi.org/10.1016/j.compedu.2011.03.009

\section{AUTOR/ES:}

\section{Santiago Tejedor}

Profesor Agregado. Director del Departamento de Periodismo y Ciencias de la Comunicación de la Universidad Autónoma de Barcelona. Doctor en Periodismo y Ciencias de la Comunicación por la UAB. Doctor en Ingeniería de Proyectos por la Universidad Politécnica de Cataluña. Miembro del Grupo de Investigación 'Gabinete de Comunicación y Educación'. Ha participado en varias investigaciones financiadas relacionadas con Media Literacy y participación ciudadana. Ha realizado estancias de investigación en Colombia, Costa Rica y Nicaragua. Posee el título honorífico "Egregius Educator", otorgado por el Consejo Superior de Dirección de la Universidad de Ciencias Comerciales de Managua y el reconocimiento como "Visitante distinguido" de la Universidad Tecnológica de Honduras. Sus líneas de investigación son: ciberperiodismo, convergencia mediática y nuevas narrativas transmedia.

santiago.tejedor@uab.cat

\section{Índice H: 14}

Orcid ID: http://orcid.org/0000-0002-5539-9800

Google Scholar: https://scholar.google.es/citations?user=693px8EAAAAJ\&hl=en

\section{Laura Cervi}

Investigadora Post-Doctoral del Departamento de Periodismo y Ciencias de la Comunicación de la UAB. Doctora en Ciencia Política por la Universitá di Pavia, Italia y por la UAB. Miembro del Grupo de Investigación 'Gabinete de Comunicación y Educación'. Ha participado en varias investigaciones financiadas en convocatorias públicas relacionadas con Media Literacy y participación ciudadana: "DINAMIC, Desarrollo de indicadores de alfabetización mediática individuales, corporativos y ciudadanos" (2012-2014); "Showing films and other audio-visual content in European Schools - 
Obstacles and best practices" (2013-2014); "EMEDUS, European Media Literacy Education Study" (2012-2014). Ha sido, junto con el Dr. José Manuel Perez Tornero, Investigadora Principal del proyecto europeo Y-NEX, European Youth News Exchange. En el 2014 fue investigadora visitante en la Newberry Library de Chicago (EEUU).

laura.cervi@uab.cat

Índice H: 2

Orcid ID: www.orcid.org/0000-0002-0376-0609

Google Scholar: https://scholar.google.es/citations?user=i-cg6lUAAAAJ\&hl=en

\section{Fernanda Tusa}

Docente investigadora. Doctora en Comunicación Social por la Universidad Austral de Argentina. Miembro del Grupo de Investigación en Discurso, Comunicación y Web 'GIDCOWEB' de la Universidad Técnica de Machala (UTMACH, Ecuador), Miembro del Proyecto de Investigación 'Las buenas prácticas pedagógicas de los edutubers' de la UTMACH. Forma parte del equipo de acompañamiento editorial de la Dirección de Investigación de la UTMACH. Miembro de la Red Internacional de Investigación de la Gestión de la Comunicación, XESCOM. Miembro de la Red Interuniversitaria de Investigación sobre Competencias Mediáticas para la Ciudadanía, ALFAMED. Ha sido becaria de postgrado de CLACSO y Fundación Ceibal. Sus líneas de investigación se centran en nuevos lenguajes digitales, arte digital narrativas híbridas, sociología de la tecnocultura y fenómenos sociales emergentes relacionados con la tecnología.

ftusa@utmachala.edu.ec

Índice H: 4

Orcid ID: https://orcid.org/0000-0002-1570-9579

Google Scholar: https://scholar.google.es/citations?user=EVNfD98AAAAJ\&hl=es\&oi=ao

\section{Alberto Parola}

Profesor Asociado de Pedagogía Experimental, Departamento de Filosofía y Ciencias de la Educación de la Universidad de Turín y psicólogo. Intereses científicos: investigación educativa, educación en medios, innovación didáctica, tecnologías de aprendizaje. Es Presidente de la Cooperativa Egò (Medios y DSA) y Presidente del Centro de Investigación CinEduMedia. Fue consultor científico de MIUR (Ministerio de Educación), director educativo de ExtracampusTv, creador y coordinador del proyecto europeo "On Air" y vicepresidente de MED (Educación en medios). Realizó consultoría científica para Rai-Radio Televisión Italiana y formó parte del grupo de expertos del proyecto europeo Emedus. Formó parte de la gestión de la Sociedad de Aprendizaje y Educación informada para Evidencia (SAPIE). Ha realizado numerosos informes en escuelas, convenciones, reuniones y conferencias a nivel nacional y 12 a nivel internacional (China, Brasil, Sudáfrica, Suecia, España y proyectos educativos y de investigación en Haití y Ruanda).

alberto.parola@unito.it

Indice H: 8

Orcid ID: https://orcid.org/0000-0003-0639-7777 
